This item was submitted to Loughborough's Research Repository by the author.

Items in Figshare are protected by copyright, with all rights reserved, unless otherwise indicated.

\title{
Ultrafast electronic energy transfer in an orthogonal molecular dyad
}

PLEASE CITE THE PUBLISHED VERSION

https://doi.org/10.1021/acs.jpclett.7b00089

\section{PUBLISHER}

() American Chemical Society (ACS)

\section{VERSION}

AM (Accepted Manuscript)

\section{PUBLISHER STATEMENT}

This work is made available according to the conditions of the Creative Commons Attribution-NonCommercialNoDerivatives 4.0 International (CC BY-NC-ND 4.0) licence. Full details of this licence are available at: https://creativecommons.org/licenses/by-nc-nd/4.0/

\section{LICENCE}

CC BY-NC-ND 4.0

\section{REPOSITORY RECORD}

Wiebeler, Christian, Felix Plasser, Gordon J. Hedley, Arvydas Ruseckas, Ifor D. W. Samuel, and Stefan Schumacher. 2019. "Ultrafast Electronic Energy Transfer in an Orthogonal Molecular Dyad". figshare. https://hdl.handle.net/2134/32238. 


\title{
Ultrafast Electronic Energy Transfer in an Orthogonal Molecular Dyad
}

\author{
Christian Wiebeler, ${ }^{1, *}$ Felix Plasser, ${ }^{2}$ Gordon J. Hedley, ${ }^{3,4}$ \\ Arvydas Ruseckas, ${ }^{3}$ Ifor D. W. Samuel, ${ }^{3}$ and Stefan Schumacher ${ }^{1}$ \\ ${ }^{1}$ Physics Department and Center for Optoelectronics and Photonics Paderborn (CeOPP), \\ Universität Paderborn, Warburger Strasse 100, 33098 Paderborn, Germany \\ ${ }^{2}$ Institute for Theoretical Chemistry, \\ Faculty of Chemistry, University of Vienna, \\ Währingerstr. 17, 1090 Vienna, Austria \\ ${ }^{3}$ Organic Semiconductor Centre, SUPA, \\ School of Physics and Astronomy, University of St Andrews, \\ North Haugh, St Andrews, Fife, KY16 9SS, U.K. \\ ${ }^{4}$ Institut für Experimentelle und Angewandte Physik, \\ Universität Regensburg, Universitätsstrasse 31, 93053 Regensburg, Germany
}

(Dated: February 14, 2017) 
ABSTRACT: Understanding electronic energy transfer (EET) is an important ingredient in the development of artificial photosynthetic systems and photovoltaic technologies. Although EET is at the heart of these applications and crucially influences their light-harvesting efficiency, the nature of EET over short distances for covalently bound donor and acceptor units is often not well understood. Here we investigate EET in an orthogonal molecular dyad (BODT4) in which simple models fail to explain the very origin of EET. Based on nonadiabatic ab initio molecular dynamics calculations and fluorescence depolarization experiments we gain detailed microscopic insights into the ultrafast electro-vibrational dynamics following photoexcitation. Our analysis offers molecular-level insights into these processes and reveals that it takes place on timescales $\lesssim 100$ fs and occurs through an intermediate charge-transfer state.

\section{TOC Graphic:}

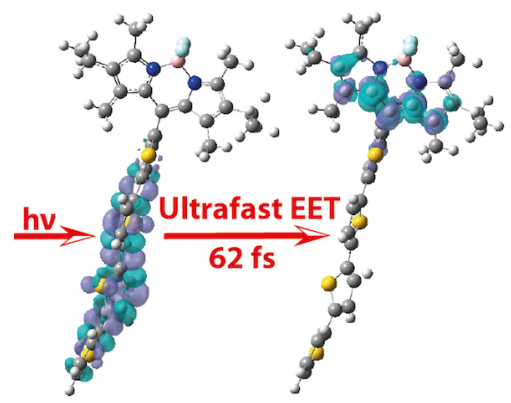

Keywords: Photophysics, Spectrum Simulation, Ultrafast Dynamics, Time-dependent DFT, Trajectory Surface Hopping 
Ultrafast relaxation dynamics following photoexcitation and electronic energy transfer (EET) in molecular systems are not only of fundamental interest ${ }^{1}$ but are of significance in light harvesting arrays, ${ }^{2,3}$ in OLEDs, ${ }^{4,5}$ and in organic photovoltaics. ${ }^{6-8}$ Förster theory, which requires the calculation of point- or line-dipole interactions, can be used to describe longrange EET. ${ }^{9,10}$ However, if donor and acceptor are too close, the theory does not hold. ${ }^{11-13}$ For specific cases, the rate of EET at short distances can be explained by hybrid approaches based on Redfield theory. ${ }^{14}$ In these approaches, the rate of EET is influenced by the spectral overlap and electronic coupling between donor and acceptor and the relative orientation of transition dipoles.

Here we study EET in an orthogonal molecular dyad (BODT4). ${ }^{15}$ In this system with orthogonal orientation between acceptor and donor unit and strong electronic coupling, simplified models as discussed above are not applicable to understand the origin or characteristics of the EET following photoexcitation. In our experiments, the ultrafast molecular dynamics following excitation is analyzed based on time-resolved fluorescence depolarization, a powerful tool to understand relaxation processes in molecular systems. ${ }^{16,17}$ To gain true microscopic insight into dynamics and in particular EET, nonadiabatic molecular dynamics based on trajectory surface hopping can be employed. ${ }^{18}$ For large sized systems such dynamics require semiempirical methods like OM2/MRCI and DFTB for the quantum chemical calculations ${ }^{19,20}$ that are combined with the classical propagation of the nuclei in the excited states including nonadiabatic couplings allowing switches between different electronic states. $^{21-23}$ For the system in our study, nonadiabatic ab initio molecular dynamics (NAAIMD) simulations are nowadays feasible. ${ }^{24-26}$ In our case the description of electronic states and excitations is firmly based on (time-dependent) density functional theory (TDDFT), where range-separated exchange-correlation functionals also accurately capture the nature of charge-separation over longer ranges.

The molecular dyad under investigation (BODT4) consists of tetrathiophene (T4) as donor and a boron dipyromethene (BOD) dye as an acceptor. The primary photoexcitation of BODT4 mostly occurs on the T4 unit. Previous studies of conjugated oligomers indicate that following photoexcitation ultrafast relaxation and internal conversion occurs. ${ }^{28-31}$ In this case the classical path approximation ${ }^{32}$ may become inapplicable. A NA-AIMD approach was recently applied to oligothiophenes with a main focus on the ultrafast deactivation of short oligomers ${ }^{33,34}$ and on the role of hot charge-transfer states in a bithiophene 

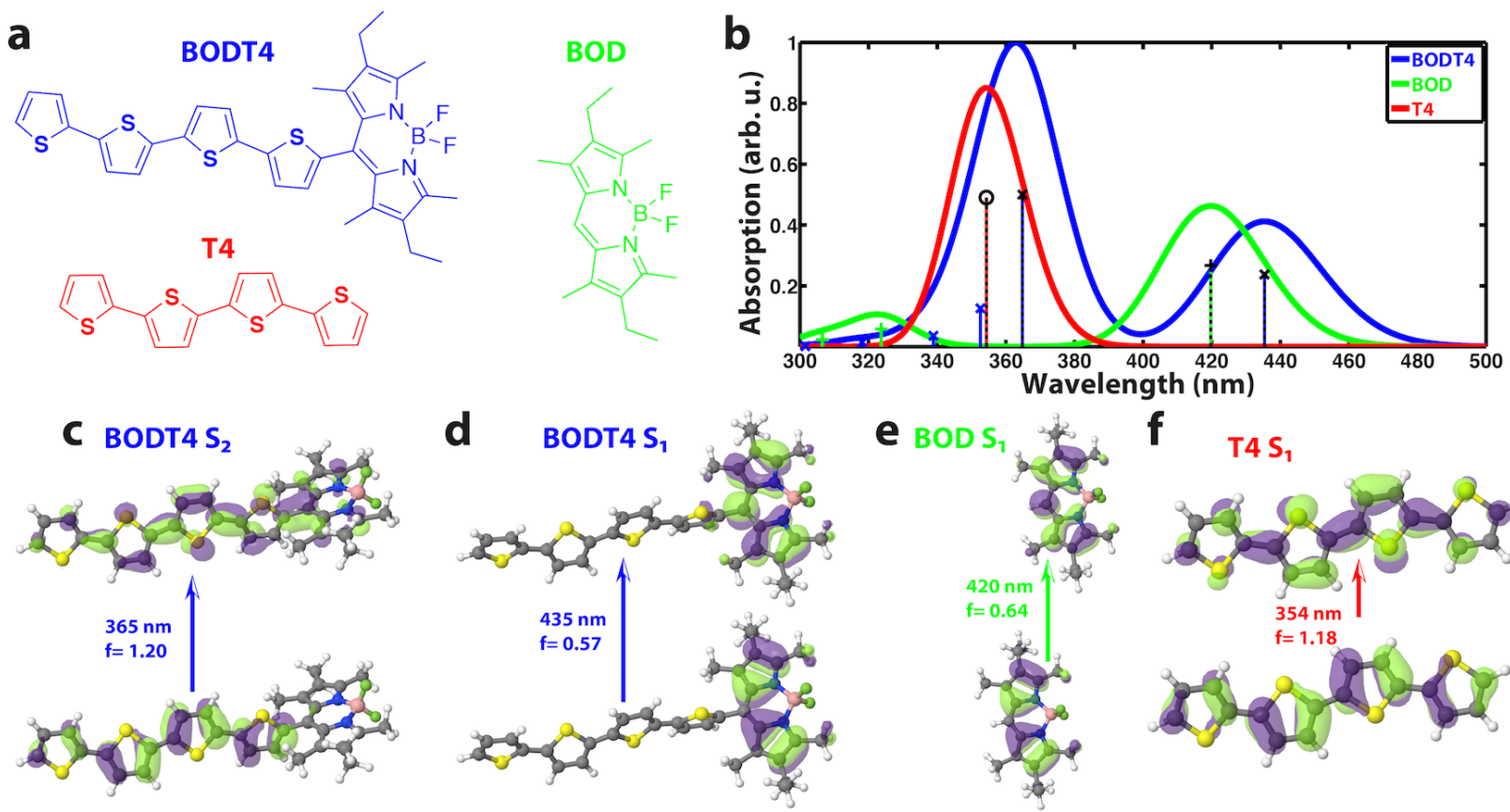

f $\mathrm{T} 4 \mathrm{~S}$

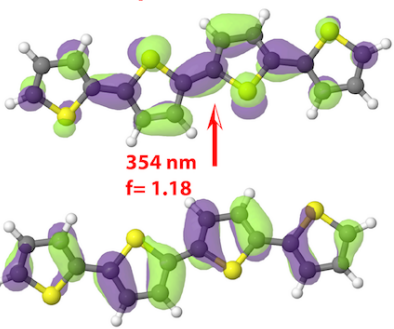

FIG. 1: (a) Sketches of the BODT4 molecular structure and its constituents BOD and T4, respectively. (b) Absorption spectra calculated for the molecules in (a). (c)-(f) Analysis of dominant excitations in terms of natural transition orbitals (NTOs) for BODT4 (c and d), BOD (e), and T4 (f). Sticks included in (b) represent wavelength and oscillator strength for each transition; transitions used for the NTO analysis are marked by black dashed lines. Each transition is dominated by one single pair of NTOs. Excitation wavelengths and oscillator strengths $f$ are given for each transition. Comparison of the transitions of BODT4 shows that the higher-energy electronic transition of BODT4 in (c) resembles the first electronic transition of the T4 molecule in (f). The lower-energy electronic transition of BODT4 in (d) resembles the first electronic transition of the BOD molecule in (e). Electronic structure calculations are based on CAM-B3LYP/6-31G*; Jmol was used to visualize the NTOs. ${ }^{27}$

dimer, respectively. ${ }^{35}$ The electron transfer from polythiophene to a carbon nanotube and corresponding hole transfer was studied based on time-dependent Kohn-Sham theory. ${ }^{36}$ In the present study we use NA-AIMD to understand the microscopic details of relaxation dynamics and EET of the primary photoexcitation to the BOD unit of BODT4. We calculate excited electronic states based on TDDFT and state specific gradients capture the photoinduced dynamics. While important information on the EET can be deduced from fluorescence 
depolarization experiments alone, ${ }^{15}$ these do not directly probe molecular structure or electronic states involved. Our theoretical calculations confirm the very efficient EET reported for BODT4 with transfer times of $\lesssim 100 \mathrm{fs}$ and reveal the involvement of an intermediate state with charge-transfer character in the transfer process. This notion may be important to inform future design and application of artificial systems with fast and efficient EET.

The focus of the present study lies on the dynamical evolution of BODT4 following photo excitation. However, before we can move on analyzing dynamical properties, a solid understanding of the static electronic properties of the system needs to be obtained. In experiment, the absorption of BODT4 exhibits two prominent absorption peaks in the visible spectral range (cf. Ref. 15 and inset of Fig. 3). These features can be used to assess the reliability of the employed quantum chemical method for the computational description of the system. We find satisfactory agreement of the absorption spectra of the BODT4 molecule and its constituents, BOD and T4, calculated using (time dependent) density functional theory with the range separated CAM-B3LYP functional ${ }^{37-39}$ in connection with the 6-31G* basis set using Gaussian 09. ${ }^{40}$ This functional is known to provide an accurate description of extended $\pi$-conjugated systems ${ }^{41-44}$. Molecular structures and calculated spectra are shown in FIG. 1. Optimization of the BODT4 geometry results in a structure, where the plane of the thiophene ring of $\mathrm{T} 4$ that is the closest to BOD is nearly perpendicular to the corresponding plane of BOD. With the range-separated functional used, we obtain two distinct absorption peaks in the range from 300 to $500 \mathrm{~nm}$, which are both blue-shifted relative to experiment, compare FIG. 1 (b) with the inset in FIG. 3. Comparing the absorption of BODT4 with the absorption of its building blocks, the lowest energy peak can be attributed to absorption of BOD and the second peak is traced back to absorption of T4. These findings are consistent with the previously reported study on BODT4. ${ }^{15}$

The calculated excited states can be further characterized based on natural transition orbitals (NTO). ${ }^{45}$ We calculate NTOs for each transition of interest using TheoDORE. ${ }^{46,47}$ They describe an electronic excitation with a minimum number of effective single-particle orbitals. In our case each investigated transition is dominated by one pair of orbitals, see FIG. 1. So one of the orbitals can be interpreted as the orbital of the hole and the other one as the orbital of the excited electron. The lowest energy excitation of BODT4 is mainly localized at BOD, its excitation energy is slightly red-shifted compared to the lowest energy excitation of BOD and only small differences in the oscillator strengths are found. Also 


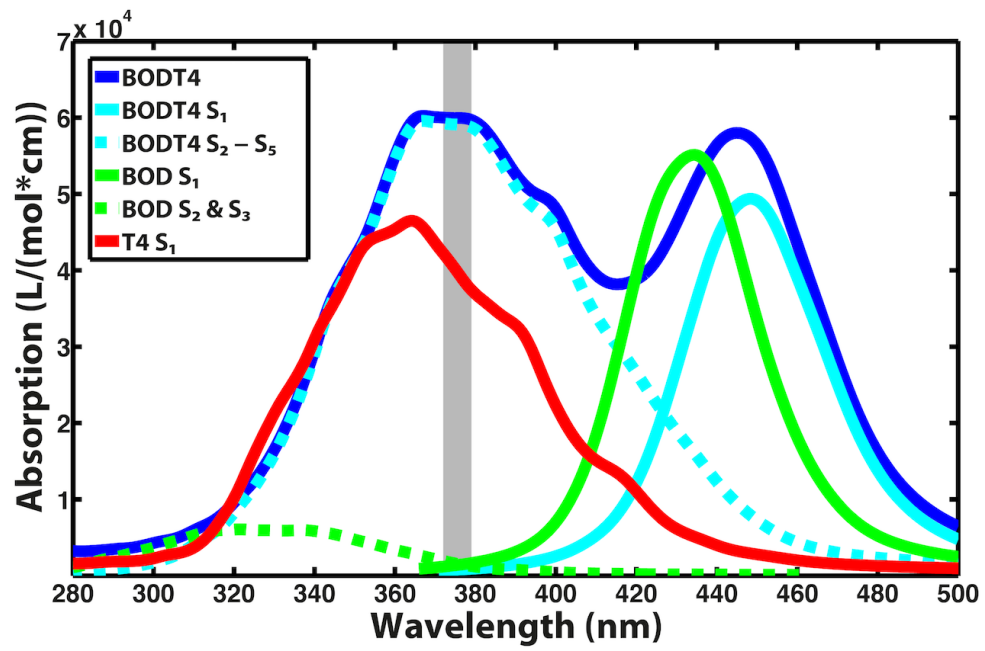

FIG. 2: Calculated absorption spectra of BODT4 (blue and cyan), BOD (green), and T4 (red) based on the nuclear-ensemble approach (NEA) using CAM-B3LYP/6-31G* for electronic structure calculations. In the spectral range shown, the only significant absorption of $\mathrm{T} 4$ can be traced back to the $\mathrm{S}_{1}$ state (solid red). For BOD, the first peak is caused by absorption of the $\mathrm{S}_{1}$ state (solid green) with an additional weaker absorption feature due to the $\mathrm{S}_{2}$ and $\mathrm{S}_{3}$ states (dashed green) between 280 and $360 \mathrm{~nm}$. For BODT4, the lower-energy peak in the spectrum is dominated by absorption into the $\mathrm{S}_{1}$ state (solid cyan). Transitions into states $\mathrm{S}_{2}$ to $\mathrm{S}_{5}$ (dashed cyan) dominate the second absorption peak at higher energies. The optical excitation window used in the calculations below is indicated in gray. It is chosen to closely resemble the experimental condition considering the slight overall shift in energy between calculated and measured absorption spectra.

the hole for the transition with the second lowest energy is mainly localized at one part of the molecule, in this case T4. In contrast to this, the excited electron is not only localized at T4, but contributions from the BOD part are also present. Nonetheless, the two bright transitions of BODT4 can be traced back to the absorption of the corresponding building blocks indicating only weak mixing of excited states.

For the measured spectra of BODT4 it is found that the higher energy peak in absorption is significantly broader than the lower one. ${ }^{15}$ However, calculating the absorption for an optimized geometry and using the same homogeneous broadening (in energy) for each electronic transition as in Fig. 1 leads to a spectrum in which the higher-energy peak is narrower (when plotted versus wavelength) than the lowest one. Much better agreement 
with the experiment in FIG. 3 (inset) is found based on spectrum simulation via nuclearensemble approach (NEA) ${ }^{48}$ interfacing Newton- ${ }^{49}$ and Gaussian $09 .{ }^{40}$ This approach takes the quantum mechanical zero point vibrations in the electronic ground state into account. It can be employed to model absorption bands more accurately than single-point calculations (though vibronic transitions are not explicitly considered). A vibrational analysis for the electronic ground state is used to generate an ensemble of 1000 geometries based on a Wigner-distribution. Subsequently, the first ten excited states for each geometry are calculated. The resulting ensemble spectrum is shown in FIG. 2. The absorption of T4 in the plotted range is dominated by the absorption of the $\mathrm{S}_{1}$ state. This state has a strong variation in excitation energies depending on the actual geometry, leading to a broad absorption band for the ensemble. The ensemble absorption into the $\mathrm{S}_{1}$ state of BOD is significantly narrower. The absolute absorption of $\mathrm{T} 4$ is in good agreement with experiment, the absolute absorption for BOD is slightly too low compared to experiment as the broadening is slightly overestimated. ${ }^{15}$ Overall, the absorption for the two peaks of BODT4 is dominated by the absorption of the corresponding building blocks. It remains similar in shape and is slightly red-shifted.

In the following we turn our attention to the ultrafast dynamics of photoluminescence (PL) emitted by BODT4 after photoexcitation. Experimental results are shown in FIG. 3 and were recorded using the fluorescence upconversion technique described previously. ${ }^{50} \mathrm{~A}$ low concentration solution of BODT4 in cyclohexane was placed in a rotating sample holder to minimize laser degradation. The sample was excited by light pulses of $100 \mathrm{fs}$ duration at a wavelength of $425 \mathrm{~nm}$ and repetition rate of $80 \mathrm{MHz}$ by frequency doubling the output of a Ti:Sapphire laser operating at $850 \mathrm{~nm}$. This ensured optical excitation of T4 with almost no excitation of any higher absorption bands of BOD. The excitation window is on the red side of the main higher-energy absorption feature. The residual $850 \mathrm{~nm}$ light acted as the gating pulse and was mixed with $600 \mathrm{~nm}$ PL from the sample in a BBO crystal to produce upconverted light at $350 \mathrm{~nm}$ that was spectrally filtered from all other wavelengths and detected on a photomultiplier tube. Scanning of an optical delay line on the gating pulse enabled temporal resolution to be obtained. The instrument response function, obtained by upconverting Raman signals from water, gave a $270 \mathrm{fs}$ full-width half-maximum Gaussian profile. $600 \mathrm{~nm}$ was chosen as the detection wavelength to ensure that only BOD PL was recorded and that there was no overlapping PL from the initially accessed T4. The obtained 


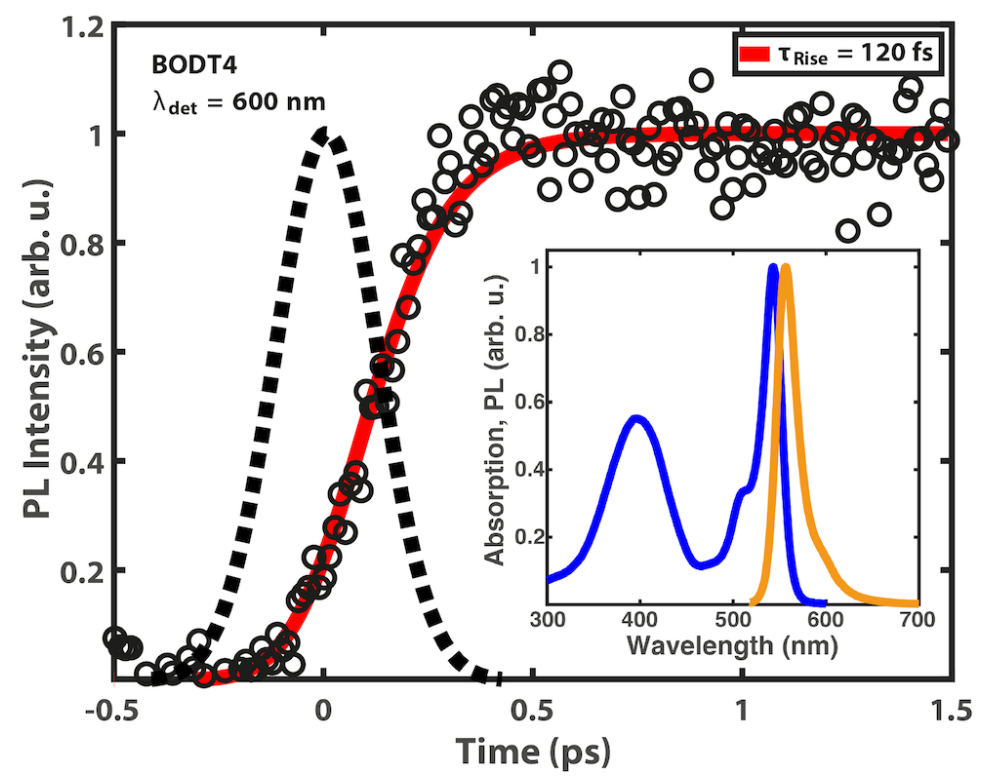

FIG. 3: Measured ultrafast photoluminescence of the BODT4 dyad (open circles), recorded after excitation at $425 \mathrm{~nm}$ (exciting only the higher-energy absorption band related to T4).

The photoluminescence is detected on the far low-energy side at $600 \mathrm{~nm}$ and is only sensitive to emission from BOD. In this configuration, the dynamics of the PL is directly related to the dynamics of the energy transfer from the initial excitation on $\mathrm{T} 4$ to the emissive state on BOD. The solid red line is a best fit to the data, giving an exponential rise-time constant of $120 \mathrm{fs}$ and a long-lived offset. The instrument response function is shown as a dotted line, and has a 270 fs full-width half-maximum. The inset shows the measured absorption (blue) and photoluminescence spectra (orange).

PL kinetics thus describe energy transfer from T4 to BOD. They can be fitted with an exponential rise-time of the form $y=-e^{-x / \tau}+1$, where the first component describes the rise-time, with time constant $\tau$, and the second component simply represents a long-lived non-decaying offset as the natural decay from BOD is on a much longer timescale than is recorded for these traces. This equation is convolved with the instrument response function and the resulting function is compared with the experimental data to produce a best fit by least squares iteration. By fitting in this way we find the data to be described well with $\tau=120 \mathrm{fs}$.

The photoluminescence and rotation of the transition dipole observed in the experiments are averages over many molecules. ${ }^{15}$ To obtain comparable results from NA-AIMD simulations a swarm of trajectories for a set of initial conditions is considered and propa- 


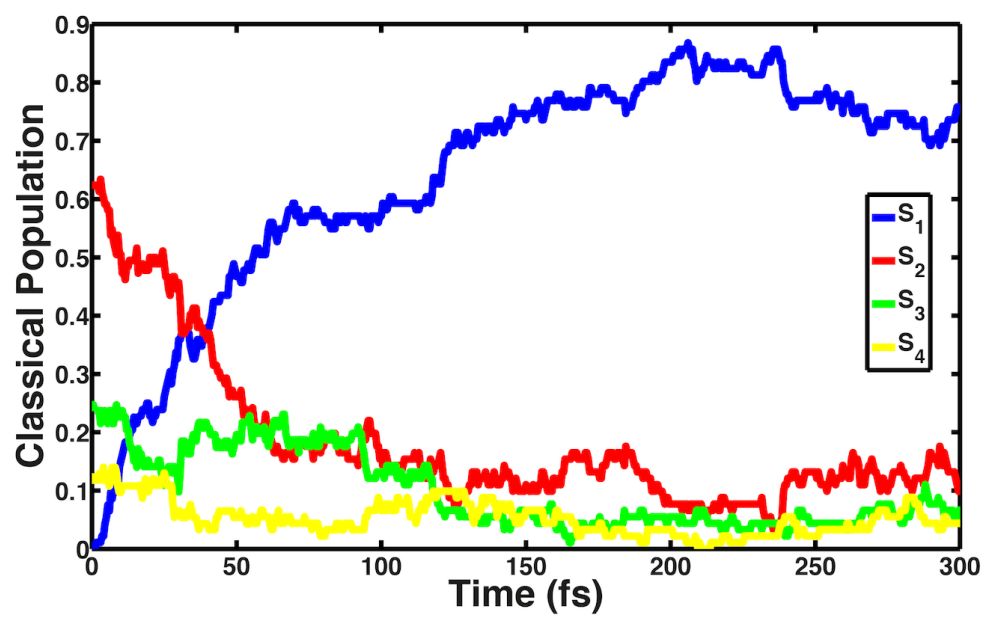

FIG. 4: Dynamics of the classical population following photoexcitation calculated based on nonadiabatic ab initio molecular dynamics using CAM-B3LYP/6-31G* for electronic structure calculations. The classical population is defined as the ratio of the trajectories in the corresponding state over the total number of trajectories. Shown are the populations for states $\mathrm{S}_{1}$ (blue), $\mathrm{S}_{2}$ (red), $\mathrm{S}_{3}$ (green), and $\mathrm{S}_{4}$ (yellow). Overall, an almost exponential decay of the higher state populations is found and relaxation to the $\mathrm{S}_{1}$ state occurs on a fast $\lesssim 100$ fs time scale.

gated in time. We perform these calculations for a microcanonical ensemble. The initial conditions are obtained for vertical excitation of the geometries that are obtained from a Wigner distribution and underlying the ensemble absorption spectrum in FIG. 2. To compute the dynamical evolution after excitation, we use trajectory surface hopping with the fewest switches criterion ${ }^{18,51}$ based on wavefunction overlap ${ }^{52}$ with decoherence correction ${ }^{53}$ by interfacing Gaussian $09^{40}$ and Newton-X. ${ }^{49,54}$ Based on the NEA spectrum of BODT4, an excitation window centered at $3.3 \mathrm{eV}$ with a width of $0.03 \mathrm{eV}$ is chosen (about 372 to $379 \mathrm{~nm}$, slightly red-shifted relative to the absorption maximum in FIG. 2). The excitation is chosen to qualitatively resemble the scenario found in the experiments (taking into account the computed excitation wavelengths, which are blue-shifted compared with experiment). The energy window is chosen in a region with minimal absorption of the BOD constituent to avoid its direct excitation. The initial states for the time evolution then are: 58 in $\mathrm{S}_{2}$, 23 in $\mathrm{S}_{3}, 11$ in $\mathrm{S}_{4}$, and 1 in $\mathrm{S}_{5}$. Overall, 93 trajectories are considered, representing all the excited states contributing significantly to the higher-energy absorption peak. Full details on methods are given in the Supporting Information. 
First we turn our attention to the average population of excited states following photoexcitation. The dynamics of these (classical) populations are shown in FIG. 4. A pronounced decay of the states $S_{2}$ to $S_{4}$ within the first $100 \mathrm{fs}$ is found and the $\mathrm{S}_{1}$ state is mainly populated. This latter state is taken as an indicator that an EET to the BOD part has taken place, so that its rise time corresponds to the rise time of the signal measured in experiment. This assumption is based on the static calculations where the $\mathrm{S}_{1}$ state is localized at BOD. However the actual character of this excited state might depend on the geometry as discussed in more detail for a sample trajectory below. After the relaxation into the $\mathrm{S}_{1}$ state, in the microcanonical ensemble without thermostat or explicit solvation, a dynamical equilibrium is reached for the excited state manifold, showing only small fluctuations of the populations of individual states. The decay of the sum of the populations of the states $\mathrm{S}_{2}$ to $\mathrm{S}_{4}$ and the rise of the $S_{1}$ state population were fitted using exponential functions with an offset of 0.2 to account for the residual population found in the higher excited states. From both fits a time constant of $62 \mathrm{fs}$ was obtained. This reproduces fairly well the experimental timescale for fluorescence de-polarization of 120 fs for BODT4 in cyclohexane solution. ${ }^{15}$ We note that the prediction of actual time constants is known to be quite sensitive to the method employed for electronic structure calculations. ${ }^{33}$ Furthermore, no coupling to the environment was included. No removal of excess energy nor interaction with surrounding solvent molecules occurs in the calculations. Both is expected to slow down the EET dynamics. We can also determine the orientation between the transition dipole of the excitation and at the end of the dynamics simulations, i.e. at $300 \mathrm{fs}$, for the current states of the trajectories. We find an average angle of $81^{\circ}$ which is also in fair agreement with the experimental value of $64^{\circ}$.

Having obtained first insights into ensemble averages and average time constants for excitation energy transfer above, the fully microscopic approach used here allows a much more detailed understanding of the dynamics and underlying processes for individual molecules. In particular analyzing the character of the electronic states involved in the dynamics will give detailed information about relevant microscopic transfer mechanisms. Therefore, we further investigate a representative trajectory showing ultrafast relaxation and EET. We note that FIG. 5 shows a particularly fast relaxation and EET but is representative for a large number of trajectories in the main features of the transfer process as discussed below. In FIG. 5 the character of the current electronic state is tracked over time by depicting excitation energy and oscillator strength. The starting point of the trajectory is an excitation 


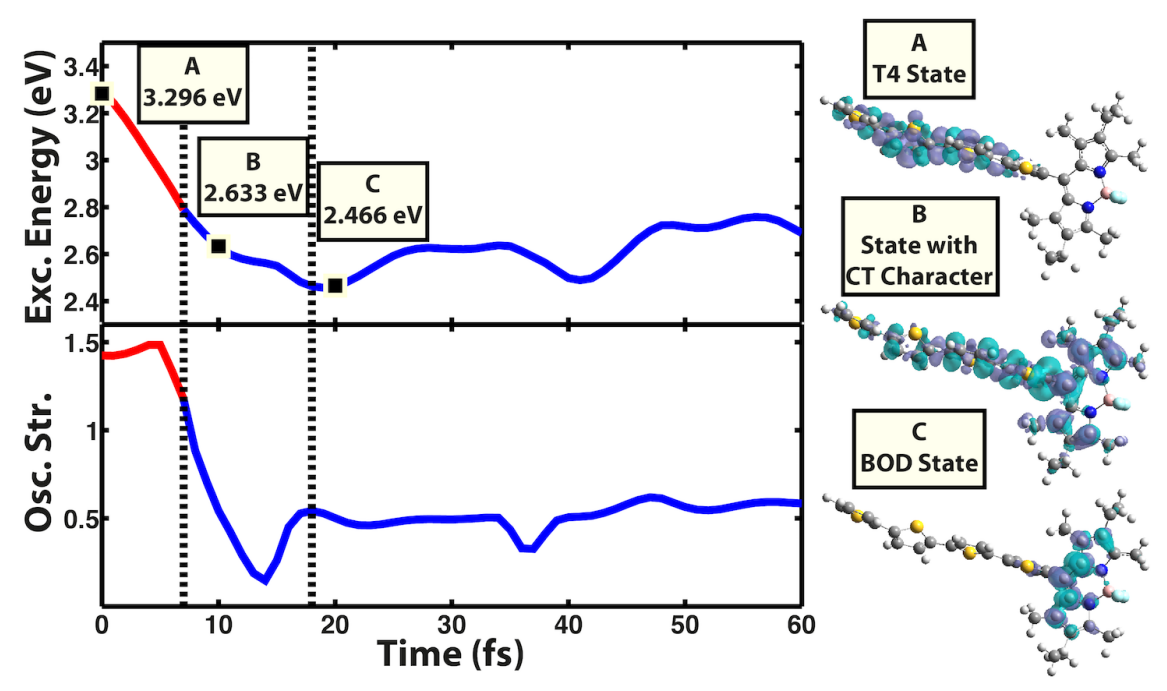

FIG. 5: Temporal evolution of the excitation energy (top) and oscillator strength (bottom) for a representative trajectory showing ultrafast excitation energy transfer. The main features are an initial lowering of the energy of the populated state, a dark intermediate state with partial charge-transfer character and a state at the end of the dynamics that resembles the first excited state of the static calculations, localized on the BOD unit. For each time, shown are the values for the current state in the dynamics calculations. Initial relaxation from the $S_{2}$ (red) to the $S_{1}$ (blue) state takes place within $7 \mathrm{fs}$. This is followed by a transfer of the excitation to the BOD unit through an intermediate state with partial charge-transfer character with low oscillator strength. Subsequently, no further energy relaxation on the BOD unit is found. On the right, electronic states involved in the dynamics are visualized based on differences of the electronic densities between the current excited state and the ground state. Initially, the excited state is localized on T4 (top), dynamical evolution and relaxation first leads to a state with charge-transfer character (middle), and eventually the excitation is completely localized on the BOD unit (bottom). CAM-B3LYP/6-31G* was used for electronic structure calculations and GaussView was used for visualization. ${ }^{55}$ Positive electron density differences (excited electron) are colored in blue and negative electron density differences (hole generation) in cyan.

localized at $\mathrm{T} 4$, which is the $\mathrm{S}_{2}$ state in this case, in which 58 of the 93 trajectories start. Within the first few fs the energy of the excited state decreases, which is found for about $88 \%$ of all trajectories, and the excitation delocalizes: the electron is delocalized over both the BOD and T4 unit while the hole remains at T4. This is accompanied by a lowering of 
the oscillator strength to nearly zero as found in $80 \%$ of the computed trajectories. Upon relaxation into the first excited state, electron and hole are still separated in space. After about $10 \mathrm{fs}$ of dynamics in the hot $\mathrm{S}_{1}$ state, $\mathrm{S}_{1}$ and $\mathrm{S}_{2}$ are still close in energy and population is repeatedly transferred between the two states, $c f$. FIG. S9. Afterwards, the $\mathrm{S}_{1}$ state localizes at BOD and after about $20 \mathrm{fs}$, the characteristics of the current excited state are similar to the first excited state obtained for BODT4 reported in FIG. 1, i.e. excitation energy between 2.5 and $3.0 \mathrm{eV}$ and an oscillator strength of about 0.5. These two typical features are found at the end of $60 \%$ of all computed trajectories. For the remaining trajectories, similar behavior is still found but is accompanied by stronger fluctuations of the oscillator strength and hoppings back into higher excited states. The latter could potentially be eliminated dissipating excess energy to the environment. We find no signature of a hot excited state on the BOD unit during or after the EET, in agreement with conclusions drawn from experiment. ${ }^{15}$ In contrast to this, the relaxation through the excited states is mediated by a state with charge-transfer character. We note that the energy alone is not an appropriate measure for the character of an excited state, because the excitation energies strongly depend on the molecular geometry and the energetic ordering of excitations changes during the dynamics. We observe that for the sample trajectory shown, first relaxation into the first excited state occurs, directly followed by transfer of the excitation. As a similar behavior is found for many trajectories, fitting the rise of the $\mathrm{S}_{1}$ state population might lead to a slight underestimation of the actual EET time. To characterize the temporal evolution of the excited states further, the position of the excitation ${ }^{47}$ and the size of the corresponding exciton ${ }^{56}$ can be investigated. These results are reported in FIG. S10 of the Supporting Information for the sample trajectory discussed. This figure highlights the charge-transfer character of the intermediate state but also shows that the charge separation is never complete, i.e. there is a strong mixing between local and charge-transfer states. We note that discussion and nomenclature of states on such fast timescales can be challenging, and thus the mixing of local and charge transfer states will lead to a complex picture not easily characterised by simple terminology, such as calling a state "charge transfer". None the less, the calculations clearly reveal that purely local states cannot account for the EET observed.

In this study, we investigated the absorption and excitation energy transfer mechanisms in BODT4 on a microscopic level using state-of-the-art quantum chemical methods. Our investigations demonstrate that the different broadenings for the two absorption peaks of 
BODT4 can be traced back to the absorption of the corresponding building blocks and the broad structure of the second peak is caused by the structural flexibility of the T4 part of the BODT4 molecule. Based on non adiabatic ab-initio molecular dynamics (NA-AIMD) simulations, we find that ultrafast relaxation and excitation energy transfer occurs on a time scale of $62 \mathrm{fs}$, in fair agreement with experimental findings based on fluorescence depolarization. On a microscopic level, we find that EET is mediated by an intermediate state with charge-transfer character after ultrafast relaxation of the initial excitation on the T4 unit. The important role a charge-transfer state plays in the ultrafast EET observed in the present study could also inform attempts realizing artificial systems showing efficient EET. The same theoretical approach used here would also allow detailed studies on EET in related molecular dyads of slightly larger size, ${ }^{57}$ and of further ultrafast photophysical processes in molecular dyads that are for example composed of BOD as fluorophore and of diarylethenes as photoswitch. ${ }^{58,59}$ This could elucidate the role of different underlying light-induced processes on a molecular level.

\section{Associated content}

\section{Supporting Information}

Description and further information regarding the methods for spectrum calculation (Section A), spectrum simulation using the nuclear-ensemble approach (Section B), and nonadiabatic dynamics simulations (Section C); further analysis of the trajectory shown (Section D), and a full author list of Reference [40] (Section E). An animation of the trajectory analyzed in FIG. 5 is included in the online material.

\section{Author information}

\section{Corresponding author}

stefan.schumacher@uni-paderborn.de

\section{Notes}

The authors declare no competing financial interest. 


\section{Acknowledgements}

C.W. and S.S. thank the German Research Foundation (DFG: GRK 1464) for financial support and $\mathrm{PC}^{2}$ for computing time. S.S. acknowledges support through the Heisenberg programme of the DFG. F.P. acknowledges support by the VSC Research Center funded by the Austrian Federal Ministry of Science, Research, and Economy (bmwfw). The St Andrews group acknowledges support from the European Research Council (grant number 321305) and the Engineering and Physical Sciences Research Council (grant EP/L017008/1). I.D.W.S. also acknowledges support from a Royal Society Wolfson Research Merit Award.

* Present address: Fritz Haber Center for Molecular Dynamics Research, Institute of Chemistry, The Hebrew University of Jerusalem, Jerusalem 91904, Israel

1 May, V.; Kühn, O. Charge and Energy Transfer Dynamics in Molecular Systems; Wiley-VCH Verlag, 2011.

2 Engel, G. S.; Calhoun, T. R.; Read, E. L.; Ahn, T.-K.; Mančal, T.; Cheng, Y.-C.; Blankenship, R. E.; Fleming, G. R. Evidence for wavelike energy transfer through quantum coherence in photosynthetic systems. Nature 2007, 446, 782-786.

3 Mirkovic, T.; Ostroumov, E. E.; Anna, J. M.; van Grondelle, R.; Govindjee,; Scholes, G. D. Light Absorption and Energy Transfer in the Antenna Complexes of Photosynthetic Organisms. Chem. Rev. 2017, 117, 249-293.

4 Ingram, G. L.; Nguyen, C.; Lu, Z.-H. Long-Range Energy Transfer and Singlet-Exciton Migration in Working Organic Light Emitting Diodes. Phys. Rev. Applied 2016, 5, 064002.

5 Vollbrecht, J.; Wiebeler, C.; Neuba, A.; Bock, H.; Schumacher, S.; Kitzerow, H. Bay-Extended, Distorted Perylene Esters Showing Visible Luminescence after Ultraviolet Excitation: Photophysical and Electrochemical Analysis. J. Phys. Chem. C 2016, 120, 7839-7848.

${ }^{6}$ Luhman, W. A.; Holmes, R. J. Investigation of Energy Transfer in Organic Photovoltaic Cells and Impact on Exciton Diffusion Length Measurements. Adv. Funct. Mater. 2011, 21, 764-771.

7 Shaw, P. E.; Ruseckas, A.; Samuel, I. D. W. Distance dependence of excitation energy transfer between spacer-seperated conjugated polymer films. Phys. Rev. B 2008, 78, 245201.

8 Tautz, R.; Da Como, E.; Wiebeler, C.; Soavi, G.; Dumsch, I.; Fröhlich, N.; Grancini, G.; 
Allard, S.; Scherf, U.; Cerullo, G. et al. Charge Photogeneration in Donor-Acceptor Conjugated Materials: Influence of Excess Excitation Energy and Chain Length. J. Am. Chem. Soc. 2013, $135,4282-4290$.

9 Wu, P.; Brand, L. Resonance Energy Transfer: Methods and Applications. Anal. Biochem. 1994, 218, 1-13.

10 Denis, J.-C.; Schumacher, S.; Hedley, G. J.; Ruseckas, A.; Morawska, P. O.; Wang, Y.; Allard, S.; Scherf, U.; Turnbull, G. A.; Samuel, I. D. W. et al. Subpicosecond Exciton Dynamics in Polyfluorene Films from Experiment and Microscopic Theory. J. Phys. Chem. C 2015, 119, 9734-9744.

11 Scholes, G. D. Long-Range Resonance Energy Transfer in Molecular Systems. Annu. Rev. Phys. Chem. 2003, 54, 57-87.

12 Renger, T. Theory of excitation energy transfer: from structure to function. Photosynth. Res. 2009, 102, 471-485.

13 Denis, J.-C.; Schumacher, S.; Galbraith, I. Quantitative description of interactions between linear organic chromophores. J. Chem. Phys. 2012, 137, 224102.

14 Ishizaki, A.; Fleming, G. R. On the adequacy of the Redfield equation and related approaches to the study of quantum dynamics in electronic energy transfer. J. Chem. Phys. 2009, 130, 234110.

15 Hedley, G. J.; Ruseckas, A.; Benniston, A. C.; Harriman, A.; Samuel, I. D. W. Ultrafast Electronic Energy Transfer Beyond the Weak Coupling Limit in a Proximal but Orthogonal Molecular Dyad. J. Phys. Chem. A 2015, 119, 12665-12671.

16 Montgomery, N. A.; Denis, J.-C.; Schumacher, S.; Ruseckas, A.; Skabara, P. J.; Kanibolotsky, A.; Paterson, M. J.; Galbraith, I.; Turnbull, G. A.; Samuel, I. D. W. Optical Excitations in StarShaped Fluorene Molecules. J. Phys. Chem. A 2011, 115, 2913-2919.

17 Montgomery, N. A.; Hedley, G. J.; Ruseckas, A.; Denis, J.-C.; Schumacher, S.; Kanibolotsky, A. L.; Skabara, P. J.; Galbraith, I.; Turnbull, G.; Samuel, I. D. W. Dynamics of fluorescence depolarisation in star-shaped oligofluorene-truxene molecules. Phys. Chem. Chem. Phys. 2012, $14,9176-9184$.

18 Tully, J. C. Molecular dynamics with electronic transitions. J. Chem. Phys. 1990, 93, 10611071.

19 Thiel, W. Semiempirical quantum-chemical methods. WIREs: Comp. Mol. Sci. 2014, 4, 145- 
157.

20 Pal, S.; Trivedi, D. J.; Akimov, A. V.; Aradi, B.; Frauenheim, T.; Prezhdo, O. V. Nonadiabatic Molecular Dynamics for Thousand Atom Systems: A Tight-Binding Approach toward PYXAID. J. Chem. Theory Comput. 2016, 12, 1436-1448.

21 Fabiano, E.; Lan, Z.; Lu, Y.; Thiel, W. In Conical Intersections. Theory, Computation and Experiment; Domcke, W., Yarkony, D. R., Köppel, H., Eds.; Advanced Series in Physical Chemistry; World Scientific Publishing, 2011; Vol. 17; Chapter 12, pp 463-496.

22 Nelson, T.; Fernandez-Alberti, S.; Roitberg, A. E.; Tretiak, S. Nonadiabatic Excited-State Molecular Dynamics: Modeling Photophysics in Organic Conjugated Materials. Acc. Chem. Res. 2014, 47, 1155-1164.

23 Ondarse-Alvarez, D.; Oldani, N.; Tretiak, S.; Fernandez-Alberti, S. Computational Study of Photoexcited Dynamics in Bichromophoric Cross-Shaped Oligofluorene. J. Phys. Chem. A 2014, 118, 10742-10753.

24 Plasser, F.; Granucci, G.; Pittner, J.; Barbatti, M.; Persico, M.; Lischka, H. Surface hopping dynamics using a locally diabatic formalism: Charge transfer in the ethylene dimer cation and excited state dynamics in the 2-pyridone dimer. J. Chem. Phys. 2012, 137, 22A514.

25 Curchod, B. F. E.; Rothlisberger, U.; Tavernelli, I. Trajectory-Based Nonadiabatic Dynamics with Time-Dependent Density Functional Theory. ChemPhysChem 2013, 14, 1314-1340.

26 Wang, L.; Long, R.; Prezhdo, O. V. Time-Domain Ab Initio Modeling of Photoinduced Dynamics at Nanoscale Interfaces. Annu. Rev. Phys. Chem. 2015, 66, 549-579.

27 Jmol: an open-source Java viewer for chemical structures in 3D. http://www . jmol .org/.

28 Banerji, N.; Cowan, S.; Vauthey, E.; Heeger, A. J. Ultrafast Relaxation of the Poly(3hexylthiophene) Emission Spectrum. J. Phys. Chem. C 2011, 115, 9726-9739.

29 Fazzi, D.; Grancini, G.; Maiuri, M.; Brida, D.; Cerullo, G.; Lanzani, G. Ultrafast internal conversion in a low band gap polymer for photovoltaics: experimental and theoretical study. Phys. Chem. Chem. Phys. 2012, 14, 6367-6374.

30 Clark, J.; Nelson, T.; Tretiak, S.; Cirmi, G.; Lanzani, G. Femtosecond torsional relaxation. Nat. Phys. 2012, 8, 225-231.

31 Grancini, G.; Maiuri, M.; Fazzi, D.; Petrozza, A.; Egelhaaf, H.-J.; Brida, D.; Cerullo, G.; Lanzani, G. Hot exciton dissociation in polymer solar cells. Nat. Mater. 2013, 12, 29-33.

32 Akimov, A. V.; Prezhdo, O. V. The PYXAID Program for Non-Adiabatic Molecular Dynamics 
in Condensed Matter Systems. J. Chem. Theory Comput. 2013, 9, 4959-4972.

33 Fazzi, D.; Barbatti, M.; Thiel, W. Modeling ultrafast exciton deactivation in oligothiophenes via nonadiabatic dynamics. Phys. Chem. Chem. Phys. 2015, 17, 7787-7799.

34 Prlj, A.; Curchod, B. F. E.; Corminboeuf, C. Excited state dynamics of thiophene and bithiophene: new insights into theoretically challenging systems. Phys. Chem. Chem. Phys. 2015, 17, 14719-14730.

35 Fazzi, D.; Barbatti, M.; Thiel, W. Unveiling the Role of Hot Charge-Transfer States in Molecular Aggregates via Nonadiabatic Dynamics. J. Am. Chem. Soc. 2016, 138, 4502-4511.

36 Long, R.; Prezhdo, O. V. Asymmetry in the Electron and Hole Transfer at a Polymer-Carbon Nanotube Heterojunction. Nano Lett. 2014, 14, 3335-3341.

37 Lee, C.; Yang, W.; Parr, R. G. Development of the Colle-Salvetti correlation-energy formula into a functional of the electron density. Phys. Rev. B 1988, 37, 785-789.

38 Becke, A. D. Density-functional thermochemistry. III. The role of exact exchange. J. Chem. Phys. 1993, 98, 5648-5652.

39 Yanai, T.; Tew, D. P.; Handy, N. C. A new hybrid exchange-correlation functional using the Coulomb-attenuating method (CAM-B3LYP). Chem. Phys. Lett. 2004, 393, 51-57.

40 Frisch, M. J.; Trucks, G. W.; Schlegel, H. B.; Scuseria, G. E.; Robb, M. A.; Cheeseman, J. R.; Scalmani, G.; Barone, V.; Mennucci, B.; Petersson, G. A. et al. Gaussian 09 Revision D.01. Gaussian Inc. Wallingford CT 2009.

41 Jacquemin, D.; Perpète, E. A.; Scalmani, G.; Frisch, M. J.; Kobayashi, R.; Adamo, C. Assessment of the efficiency of long-range corrected functionals for some properties of large compounds. J. Chem. Phys. 2007, 126, 144105.

42 Limacher, P. A.; Mikkelsen, K. V.; Lüthi, H. P. On the accurate calculation of polarizabilities and second hyperpolarizabilities of polyacetylene oligomer chains using the CAM-B3LYP density functional. J. Chem. Phys. 2009, 130, 194114.

43 Wiebeler, C.; Tautz, R.; Feldmann, J.; von Hauff, E.; Da Como, E.; Schumacher, S. Spectral Signatures of Polarons in Conjugated Co-polymers. J. Phys. Chem. B 2013, 117, 4454-4460.

44 Mewes, S. A.; Plasser, F.; Dreuw, A. Communication: Exciton analysis in time-dependent density functional theory: How functionals shape excited-state characters. J. Chem. Phys. 2015, 143,171101 .

45 Martin, R. L. Natural transition orbitals. J. Chem. Phys. 2003, 118, $4775-4777$. 
46 Plasser, F. TheoDORE 1.2.2: a package for theoretical density, orbital relaxation, and exciton analysis. Available from http://theodore-qc.sourceforge.net.

47 Plasser, F.; Lischka, H. Analysis of Excitonic and Charge Transfer Interactions from Quantum Chemical Calculations. J. Chem. Theory Comput. 2012, 8, 2777-2789.

48 Crespo-Otero, R.; Barbatti, M. Spectrum simulation and decomposition with nuclear ensemble: formal derivation and application to benzene, furan and 2-phenylfuran. Theor. Chem. Acc. 2012, 131, 6 .

49 Barbatti, M.; Ruckenbauer, M.; Plasser, F.; Pittner, J.; Granucci, G.; Persico, M.; Lischka, H. Newton-X: a surface-hopping program for nonadiabatic molecular dynamics. WIREs: Comp. Mol. Sci. 2014, 4, 26-33.

50 Hedley, G. J.; Ruseckas, A.; Samuel, I. D. W. Ultrafast luminescence in $\operatorname{Ir}(\text { ppy })_{3}$. Chem. Phys. Lett. 2008, 450, 292-296.

51 Hammes-Schiffer, S.; Tully, J. C. Proton transfer in solution: Molecular dynamics with quantum transitions. J. Chem. Phys. 1994, 101, 4657-4667.

52 Pittner, J.; Lischka, H.; Barbatti, M. Optimization of mixed quantum-classical dynamics: Timederivative coupling terms and selected couplings. Chem. Phys. 2009, 356, 147-152.

53 Granucci, G.; Persico, M. Critical appraisal of the fewest switches algorithm for surface hopping. J. Chem. Phys. 2007, 126, 134114.

54 Plasser, F.; Crespo-Otero, R.; Pederzoli, M.; Pittner, J.; Lischka, H.; Barbatti, M. Surface Hopping Dynamics with Correlated Single-Reference Methods: 9H-Adenine as a Case Study. J. Chem. Theory Comput. 2014, 10, 1395-1405.

55 Dennington, R.; Keith, T.; Millam, J. GaussView Version 5.0.9. Semichem Inc. Shawnee Mission KS 2009.

56 Bäppler, S. A.; Plasser, F.; Wormit, M.; Dreuw, A. Exciton analysis of many-body wave functions: Bridging the gap between the quasiparticle and molecular orbital pictures. Phys. Rev. A 2014, 90, 052521 .

57 Sajjad, M. T.; Manousiadis, P. P.; Orofino, C.; Cortizo-Lacalle, D.; Kanibolotsky, A. L.; Rajbhandari, S.; Amarasinghe, D.; Chun, H.; Faulkner, G.; O'Brien, D. C. et al. Fluorescent Red-Emitting BODIPY Oligofluorene Star-Shaped Molecules as a Color Converter Material for Visible Light Communications. Adv. Optical Mater. 2015, 3, 536-540.

58 Moreno, J.; Schweighöfer, F.; Wachtveitl, J.; Hecht, S. Reversible Photomodulation of Electronic 
Communication in a $\pi$-Conjugated Photoswitch-Fluorophore Molecular Dyad. Chem. Eur. J. 2016, 22, 1070-1075.

59 Schweighöfer, F.; Dworak, L.; Hammer, C. A.; Gustmann, H.; Zastrow, M.; Rück-Braun, K.; Wachtveitl, J. Highly efficient modulation of FRET in an orthogonally arranged BODIPY-DTE dyad. Sci. Rep. 2016, 6, 28638. 